\title{
Verhalten des Kamphers zu Chlor, Jod und Brom;
}

vom

Professor Claus in Casan.

Prof. Claus in Casan prüfte das Verhalten des Kamphers zu Chlor, Jod und Brom.

$\mathrm{Zu}$ den Elementaranalysen wählte der Verf. das Verfahren von Hefs welches ihm genanere Resultate gab, als das Liebig'sche. Er bediente sich jedoch eiver Porcellanröhre und zur Erhitzung des Liebig'schen Ofens. Den erhaltenen Kohlenstoffverlust hält er im Verluste des Kaliapparates und etwas unabsorbirter Kohlensäure liegend, der nicht über $\frac{1}{4} \frac{0}{0}$ betragen haben könne, doch ergiebt sich bei Berechnung der Analysen nach dem neuen Kohlenstoffatomgewichte ein Verlust von einem ganzen Procent. Die Absorptionsröhre von Dum as ist nach dem Verf. ein sehr gutes Mittel zur Beurtheilung des Ganges der Verbrennung. Ist die Verbrennung nämlich vollständig, so bleibt đảas mit Schwefelsäure befeuchtete Bimsteinpulver unverändert, bei der geringsten Spur unverbrannter Producte färbt sich der vordere Theil roth.

\section{Verhalten des Kamphers zum Chlor.}

Es wurden Flaschen mit trocknem Chlorgase angefüllt, in diese zerriebener Kampher gethan und so dem directen Sonnenlichte ausgesetzt. Nach Verlauf von einigen Wochen warde der Kampher herausgenommen, er war etwas gelblich gefärbt, hatte ein wenig Chlor absorbirt, jedoch sonst keine wesentliche Veränderung erlitten. Wendet man sehr feuchtes Chlorgas an, so bildet sich Salzsäure und ein Theil des Kamphers verwandelt sich in flüssigen salzs. Kampher. Man leitete trocknes Chlorgas durch schmelzenden Kampher ( $170^{\circ} \mathrm{C}$.). Hierbei verflüchtigte sich der Kampher ohne bemerkbare Zersetzung.

Einige Chloride zersetzen jedoch den Kampher sehr 
leicht. Erhitzt man nämlich ein Gemenge von Sublimat und Kampher in einer Glasröhre, so entwiokelt sich Salzsäure, man nimmt einen terpentinähnlichen Geruch wahr und in der Retorte bleibt eine schwarzbraune Masse, welche nach dem'Ausziehen mit Alkohol Calomel und eine kohlige Substanz hinterläfst. Noch kräftiger wirkt das Antimonsuperchlorid ein. Bei gewöhnlicher Temp. wird der Kampher wenig davon afficirt, erwärmt man aber ein Gemenge beider Substanzen im Wasserbade, so beginnt mit dem Sieden des Wassers eine heftige Reaction unter starkem Aufschäumen und plötzlicher Entwicklung einer grofsen Menge Salzsäuregas. Sonst bildet sich nichts Gasförmiges und Flüchtiges. Nach Beendigung der sehr rasch erfolgten Zersetzung ist der Kampher in eine dicke rothbraune Masse verwandelt, gemengt mit Chlorantimon und Salzsäure. Sie wurde mit Wasser geschüttelt, wobei basisches Chlorantimon mit braungrauer Farbe niedergeschlagen und Salzsäure mit etwas Chlorantimon gelöst wurde. Die Lösung enthielt nichts. Organisches, sondern das Zersetzungsproduct des Kamphers befand sich in dem Niederschlage. Dieser wurde nach dem Auswaschen mit Alkohol bis zur völligen Erschöpfung extrahirt.

Die alkoholische Auflösung liefs nach dem Abdampfen ein eigenthümliches, weiches Harz zurück, das einen sehr angenehmen aromatischen, keineswegs kampherartigen Geruch und einen scharfen, kratzenden Geschmack besafs. Beim Erhitzen dieses Harzes entwickelt sich Salzsäure, es destilliren anfangs farblose, dann gefärbte, ̈̈lartige, wohlriechende, pfefferartig schmeckende Flüssigkeiten über und es bleibt zuletzt eine kohlige, voluminöse, glänzende Substanz zurück. Der Niederschlag des basischen Chlorantimons war noch grau gefärbt. Er wurde nun noch mit Aether extrahirt und eine dunkelbraune ins Grünliche schillernde Tinctur erhalten, welche nach dem Verdunsten einen andern harzartigen, schwarzbrannen Körper hinterliefs. Wird in eine alkoholische Kampherlösung Chlor geleitet, so 
erfolgt sehr rasche Zersetzung, diese beschränkt sich aber gröfstentheils auf den Alkohol, doch wird dabei auch der Kampher, wenngleich sehr langsam, angegriffen. Es ist jedoch sehr schwer, die Zersetzungsproducte des Alkohols von denen des Kamphers zu trennen. Nach vielen vergeblichen Versuchen gelang es endlich, den Kampher so zu zersetzen, dafs ohne Abscheidung von Kohlenstoff der Wasserstoff desselben stufenweise von Chlor ersetzt wurde.

In 1 Unze Phosphorsuperchlorür wurden 3 Unzen Kampher gelöst. Die Auflösung erfolgte ungemein leicht und das Ganze stellte eine farblose, ziemlich dünne Flüssigkeit dar. Durch diese warde 24 Stunden lang Chlor geleitet. Die Einwirkung war anfangs unter Erwärmung und starker Entwicklung von Salzsäure sehr energisch, doch nach Verlauf von einigen Stunden wurde sie bedeutend schwächer, so daf's man den Zersetzungsact durch Erwärmen der Flüssigkeiten unterstützen mufste. Zuletzt war die Reaction sehr schwach und das meiste Chlor ging unabsorbirt über. Hierauf wurde die Operation beendigt und der Inhalt der Retorte herausgenommen. Er stellte eine durchsichtige, wenig gelblich gefärbte Flüssigkeit dar von der Consistenz des canadischen Balsams. Um die Beimischung von Chlorphosphor zu zersetzen, wurde das Product anfangs mit Wasser, darauf mit einer Lösung von kohlensaurem Natron geschüttelt. Durch diese Operation wurde die Verbindung des Chlors mit zersetztem Kampher in eine weifse rahmähnliche, dicke Masse verwandelt, welche ein Gemenge von Chlorkampher mit Wasser ist. Zur Entfernung des Wassers wurde die Masse im Wasserbade flüssig gemacht und ein rascher Strom trockner Luft so lange hindurchgeleitet, bis sie durchsichtig wurde und keine Wasserdämpfe mehr bemerkbar waren. Diese Entwässèrung erfordert viel Zeit. In diesem Zustande bildet der Chlorkampher eine fast farblose, durchscheinende, salbenartige, ölige Substanz, von angenehm aromatischem Geruch. Der Geschmack ist kampherartig bitter, hin- 
terher scharf und kratzend. Er ist vollkommen neutral, unlöslich in Wasser, lälst sich jedoch mit einer geringen Menge desselben zu einer weifsen, rahmähnlichen Masse mischen. Alkohol und Aether lösen ihn leicht auf. Ein mit Chlorkampher getränkter Docht brennt, so lange man ihn in die Flamme der Weingeistlampe hält, verlischt aber beim Herausnehmen. Bei $+100^{\circ} \mathbf{C}$. nimmt er die Consistenz des Baumöls an; stärker erhitzt, zersetzt er sich unter Bildung von Salzsäure und verschieden gefärbter, chlorhaltiger, wohlriechender Oele. Anfangs geht ein farbloses Oel über, dann folgt ein schwach rosenroth gefärbtes, hierauf ein grünes und zuletzt ein schwarzbraunes. Endlich bleibt Kohle zurück. Der Chlorkampher verhält sich in dieser Beziehung auf ähnliche Weise, wie das Chlortereben. Charakteristisch ist das Verhalten des Chlorkamphers zum Antimonsuperchlorid. Mischt man einen Tropfen dieser Flüssiggkeit mit Chlorkampher, so wird dieser auf einige Augenblicke purpurroth, dann schön indigblau; mischt man hierauf etwas Wasser zu, so wird er grün. Der eben beschriebene Chlorkampher ist keine einfache Verbindung, sondern wie die Analyse nachgewiesen hat, ein Gemenge von 2 Chlorkampherarten mit verschiedenem Chlorgehalte.

\begin{tabular}{rrrrr} 
orgehalte. & \multicolumn{4}{c}{ Atome } \\
C. & $\mathbf{4 4 , 0 9}$ & $\mathbf{4 3 , 6 0}$ & $\mathbf{2 0}$ & $\mathbf{4 1 , 5 2}$ \\
H. & $\mathbf{4 , 3 3}$ & $\mathbf{4 , 2 1}$ & $\mathbf{2 5}$ & $\mathbf{4 , 5 4}$ \\
0. & $\mathbf{4 , 9 0}$ & $\mathbf{5 , 5 1}$ & $\mathbf{2}$ & $\mathbf{5 , 8 2}$ \\
Cl. & $\mathbf{4 6 , 6 8}$ & $\mathbf{4 6 , 6 8}$ & $\mathbf{7}$ & $\mathbf{4 5 , 1 2}$ \\
\hline & $\mathbf{1 0 0 , 0 0}$ & $\mathbf{1 0 0 , 0 0}$ & & $\mathbf{1 0 0 , 0 0}$.
\end{tabular}

Also ein Gemenge aus 2 Verbindungen $=\mathrm{C}_{20} \mathbf{H}_{26}$ $\mathrm{Cl}_{6} \mathrm{O}_{2}+\mathrm{C}_{20} \mathrm{H}_{24} \mathrm{Cl}_{8} \mathrm{O}_{2}$.

Wurde diese Chlorverbindung in einer Glasröhre im Wasserbade erhitzt und dann noch längere Zeit mit Chlor behandelt, so entwickelte sich noch Salzsäure, die Zersetzung erfolgte jedoch sehr langsam und es bildete sich ein Chlorkampher, der dem früheren ganz ähnlich war, doch mehr Chlor, nämlich 51 p. C. enthielt.

Die Wasserstoffentziehung kann noch weiter getrieben werden, wenn man die durch Wärme flüssig 
gemachte Substanz sehr anhaltend mit Chlor behandelt. Man erhielt auf diese Weise einen Chlorkampher, der die Consistenz eines weifsen Wachses hatte, farblos war, sonst aber in seinen Eigenschaften mit den früher angegebenen Chlorverbindungen übereinkam. Die Analyse desselben gab folgende Resultate:

Atome

\begin{tabular}{rrrr} 
C & $\mathbf{3 4 , 8 0}$ & $\mathbf{2 0}$ & $\mathbf{3 3 , 9 0}$ \\
H & $\mathbf{3 , 1 0}$ & $\mathbf{2 0}$ & $\mathbf{2 , 7 7}$ \\
0 & $\mathbf{4 , 3 6}$ & $\mathbf{4}$ & $\mathbf{4 , 4 3}$ \\
Cl & $\mathbf{5 7 , 7 4}$ & & \\
\hline & $\mathbf{1 0 0 , 0 0}$ & & $\mathbf{1 0 0 , 0 0}$.
\end{tabular}

Verhalten des Kamphers zum Brom.

Das Brom wirkt auf den Kampher wie das Chlor, es löst zwar eine beträchtliche Menge desselben auf, allein es erfolgt eine Zersetzung. Unterwirft man diese Auflösung einer Destillation, so entwickelt sich zwar etwas Bromwasserstoffsäure, doch geht der gröfste Theil des Kamphers in Auflösung des Broms unzersetzt über und kann durch Kaliauflösung ausgeschieden werden. Man liefs eine solche Lösung von Kampher in Brom drei Monate stehen und bemerkte nach Verlauf dieser Zeit braune Krystalle in der Flüssigkeit. Beim Herausnehmen zerflossen sie sogleich an der Luft zu einer gelbbraunen Flüssigkeit, aus der das Brom mit Hinterlassung von wenig verändertem $K$ ampher verdunstete. Sie waren also eine Verbindung von Brom und Kampher. - Bromphosphor zersetzt den Kampher noch leichter als Chlorphosphor. Wenn man Kampher in Brom auflöst und sehr vorsichtig Phosphor in kleinen Antheilen hinzuthut, so entsteht eine heftige Reaction; es bildet sich viel Bromwasserstoffsäure und man erhält eine braune Flüssigkeit, aus der Wasser einen öligen Bromkampher ausscheidet, der in seinen Eigenschaften dem Chlorkampher ähnlich ist. Der Verf. glaubt, dafs der Versuch noch bessere Resultate geben werde, wenn man za dem schon gebildeten Bromphosphor Kampher und Brom hinzuthut: denn auf die früher angegebene Weise ist die Reaction zu heftig. 
Verhalten des Kamphers zum Jod. und Brom.

Das Jod wirkt anders auf den Kampher als Chlor

Gleiche Theile beider Körper wurden zusammengerieben und ein Gemenge erhalten, dafs eine braune Farbe und eine dickflüssige Consistenz hatte. Es ist eine Verbindung von Jod mit unzersetztem Kampher, welche bei gewöhnlicher Temp. an der Luft schneller verdunstet, als jeder Bestandtheil einzeln für sich. Sie ist nicht löslich in Wasser, löst sich jedoch leicht in Weingeist und Aether mit hyacinthrother Farbe. Wässrige Lösungen der Alkalien scheiden daraus den Kampher unverändert ab. Ein Gemenge aus 6 Unzen von jedem Körper wurde in einer Retorte mehre Tage lang der wechselseitigen Durchdringung überlassen und darauf aus dem Sandbade destillirt. Nach sehr schwacher Erwärmung wurde die Masse flüchtig und gerieth bei $+120^{\circ} \mathrm{C}$. in starkes Sieden, wobei sich viel Jodwasserstoffsäure, in einem geregelten Strome entwickelte und unter allmählichem Steigen des Siedepunktes bis auf $+200^{\circ}$ C. ein leichtflüssiges braunes Destillat überging. Die Destillation ward beendigt als die Retorte sich von den Dämpfen des braunen Destillats vollkommen entleert hatte and man eine schwarze, siedende Flüssigkeit in der Retorte deutlich wahrnehmen konnte. Durch diese Destillation war der Kampher gröfstentheils zersetzt worden und zwar zur Hälfte in ein flüssiges Destillat und in einen schwarzen Rückstand. Das Destillat ist eine ölartige, leichtflüssige, braune Flüssigkeit, welche Dämpfe von Jodwasserstoffs. ausstölst, einen widerlich sauren, zusammenziehenden, terpentinähnlichen Geschmack und einen eigenthümlichen etwas terpentinähnlichen Geruch besitzt. In der Ruhe sondert sie sich in zwei Schichten ab; die obere leichte Schicht, welche den beträchtlichsten Theil ausmacht, besteht aus Jod und dem flüchtigen Producte der Zersetzung des Kamphers und ist nach dieser Absonderung vollkommen neutral und nicht mehr rauchend. Die untere sehr 
geringe Schicht ist höchst concentrirte, flüssige Jodwasserstoffsäure, welche stark dampft, von überschüssigem Jod braun gefärbt ist, und eine geringe Menge unzerselzten K. aufgelöst enthält. Durchs Schütteln mit etwas Queck. silber kann der Ueberschufs des Jods entfernt und die Säure entfärbt werden.

Das Hauptproduct der Zersetzung des K. ist jedoch das eben erwähnte braune jodhaltige Oel, dessen $\mathrm{Zu}$ sammensetzung ziemlich complicirt ist, das jedoch der grörsten Menge nach aus einem eigenthümlichen sauerstofffreiem Oele, Camphin, besteht. Dieses Camphin ist nur schwach an Jod gebunden, da der grörste Theil des letztern durch Schütteln des rohen Destillationsproductes mit Quecksilber entzogen werden kann. Durch diese Operation wird das brane Oel farblos und enthält nur sehr gering'e Antheile von Jod. Schüttelt man sowohl das unreine braune, als auch das durch Quecksilber entfärbte Oel mit Thierkohle, so zeigen die Flüssigkeiten, nachdem sie sich über dem Kohlenpulver geklärt haben, bei reflectirtem Lichte einen schönen blauen Schiller, der abhängig ist von einer geringen Beimischung des Colophens, das sich gleichfalls bei der Zersetzung des Kamphers als Nebenproduct bildet.

Wird das rohe Destillationsproduct mit einer conc. Kaliauflösung behandelt, so verbindet sich fast alles Jod mit dem Kali und unreines Camphin scheidet sich mit gelblicher Farbe ab. Die Kalilösung enthält aufser dem Jod noch ein eigenthümlich electronegatives Oel gelöst, das durch Säuren abgeschieden werden kann. Dieses Oel wird neben dem Colophen in sehr geringer Menge gebildet, und ist dem Kreosot so ähnlich, dafs der Verf. es Camphokreosot nennt.

Colophen und Camphokreosot bleiben zurück, wenn das rohe jodhaltige Camphin bei $+180^{\circ} \mathrm{C}$. nochmals der Destillation unterworfen wird. Steigert man hierauf die Hitze, so geht ein braungrünes, dickes Oel über, das ein Gemenge von unreinem Colophen und Camphokreosot ist. Behandelt man dieses Oel mit starker Kali- 
lauge, so verschwindet sogleich der Kreosotgeruch, das Camphokreosot löst sich anf und ungelöst bleibt ein mildes, angenehm nach Veilchen riechendes Oel, das bei durchfallendem Lichte grünbraun, bei auffallendem aber indigblau erscheint. Es ist unreines Colophen.

Colophen. Das rohe Product wurde anfangs über Aetzkalk und zuletzt über Kalium rectificirt (dabei zerlegte sich ein ansehnlicher Theil). In diesem Zustande stellt es ein dickes gelbliches Oel dar, das einen schönen violetten Schiller hat. Löst man 2 Tropfen dieses Oels in 2 Quentchen Alkohol und giefst diese Flüssigkeit in ein Gläschen, auf dessen Boden sich etwas Thierkohle befindet, so erscheint sie beim reflectirten Lichte schön dunkelblan. Colophen hat einen sehr milden Geschmack und einen sehr angenehmen Veilchengeruch. Es ist unlöslich in Wasser und schwachem Weingeist, löslich in Alkohol, Aether, Terpentinöl, Petroleum und Camphin. Angezündet brennt es mithell euchtender, stark rufsender Flamme. Es erfordert zur Destillation eine bedeutende Hitze, daher der Siedepunkt sehr hoch ist. Camphokreosot wurde durch Destillation über Aetzkalk gereinigt. Es war gelblich gefärbt, dickfiüssig, ölartig, hatte einen, dem Kreosot ganz ähnlichen Geschmack, anfangs süfslich, dann beifsend, einen kratzenden Reiz auf der Zunge erregend. Der Geruch ist dem Kreosot sehr ähnlich. Gegen Kali verhält es sich wie Kreosot und macht auch das Eiweirs gerinnen. Der Hauptunterschied liegt im spec. Gew., es ist leichter als Wasser.

Demnach besteht das flüchtige Destillationsproduct des durch Jod zersetzten K. aus: Camphin, Colophen, Camphokreosot, Jod, Jodwasserstoffsäure und etwas nnzersetztem Kampher. Es wurde, nachdem es von Jodwasserstoffs. und von freiem Jod durch Quecksilber befreit worden, analysirt.

$$
\begin{array}{ccc}
\text { C } & 83,08 & 82,85 \\
\text { H } & 12,15 & 11,86 \\
\text { O } & - & - \\
\text { J } & 3,42 & 3,42
\end{array}
$$

Arch, d.Pharm, II. Reihe, XXX, Bds. 2. Hft. 
Man sieht aus diesen Analysen, dafs das unreine Camphin einen Kohlenwasserstoff enthält, der reicher an Wasserstoff ist, als der Kohlenwasserstoff des Kamphers, ein Umstand, der im Widerspruch mit der Wirknngsweise der Haloide steht und der darthut, dafs das Jod in seiner Reaction auf Kampher von Chlor und Brom abweicht. - Die andere Hälfte des zersetzten Kamphers bildet den Rückstand in der Retorte, nachdem alles Camphin übergegangen ist. Er ist ein zusammengesetzter harzähnlicher, schwarzer Körper, zu dessen Bezeichnung der Verf. den Namen Camphoresin vorschlägt. Er besteht aus Kohle, einem eigenthümlichen Harze mit geringen Antheilen von Camphin, Colophen, Camphokreosot und Jod. Dieses Harz ist von Ansehn dem Asphalt ähnlich, glasglänzend, sehr spröde, von muschligem Bruche, läfst sich sehr leicht zu einem schwarzen Pulver zerreiben, das dem Pulver der Holzkohle sehr ähnlich ist. Es ist geruch - and geschmacklos, nur löslich in Wasser und Weingeist, löst sich aber mit Hinterlassung von Kohle in Alkohol, Aether, Petroleum, Terpentinöl und Camphin. Die Auflösangen sind braun gefärbt und schillern grünlich. Schüttelt man die Lösung in Petroleum mit Thierkohle, so wird der Schiller blau, die Lösungen der andern Flüssigkeiten aber geben, bei äbnlicher Behandlungsweise, einen schönen, hellgrünen Schiller. Das Camphoresin schmilzt sehr leicht, geräth in schwaches Sieden, stöfst dabei weifse nach Kreosot riechende Dämpfe aus, entzündet sich, brennt mit hellleuchtender Flamme und hinterlärst eine voluminöse, glänzende Kohle, welche beim Glühen die Flamme der Weingeistlampe intensiv grün färbt (Gegenwart des Jods andeutend). Bei der trocknen Destillation giebt es anfangs etwas Camphin, Camphokreosot und Colophen, dann folgt Oelgas und ein grünes weiches Harz und zuletzt bleibt Kohle garück.

Das Camphoresin wurde analysirt: 


\begin{tabular}{lrr} 
C & 88,30 & 88,85 \\
H & 8,46 & 8,33 \\
0 & $\mathbf{0 , 6 4}$ & 0,82 \\
$\mathrm{~J}$ & $2,-$ & \multicolumn{2}{c}{,-} \\
\hline & $\mathbf{1 0 0 , 0 0}$ & $\mathbf{1 0 0 , 0 0 .}$
\end{tabular}

Es ergiebt sich aus der Zusammenstellung der Resultate, dafs der Kampher bei der Zersetzung durch Jod auf folgende Weise verändert wird. Ein Theil seines Sauerstoffs bildet mit dem aequivalenten Theil Wasserstoff Wasser, in dem flüssiger Jodwasserstoff vorhanden. Es bildet sich ein Kohlenwasserstoff, der reicher an Wasserstoff ist, als das Radikal des $K$. und der in Verbindung mit Jod als jodhaltiges Camphin überdestillirt; dabei scheidet sich Kohle aus, welche in Verbindung mit einem andern Kohlenwasserstoffe, der reicher an Kohlenstoff ist als das Radikal des K., das Camphoresin bildet. Dieses entstand aus dem Camphor durch Wasserstoffentziehung mittelst Jods, welches letztere Jodwasserstoffs. bildete. Der andere Theil des Sauerstoffs bildet höchst wahrscheinlich das Camphokreosot. Das Colophen ist eine polymere Form des Radikals des Kamphers.

Camphin. Um diese Substanz aus dem rohen Destillate zu isoliren, verfährt man also: man unterwirft es einer neuen Destillation, um eine mögliche Beimengung unzersetzten Kamphers zu beseitigen. Das Destillat wird nun mit einer starken Kalilange behandelt, das. sich abscheidende Oel, unreines Camphin, gesammelt und ein paar Mal über Aetzkalk, der zuvor mit Kali gemischt worden, rectificirt. Man erhält hierbei ein vollkommen farbloses Oel, das noch Spuren von Jod enthält. Man lärst es einige Tage über Kalium stehen und destillirt es davon ab. Sollte es noch nicht vollkommen rein sein, so wiederholt man noch einmal die Destillation über Kalium. Die Reinheit der Substanz erkennt man an dem Verhalten derselben zum Kalium, erhitzt man nämlich das Camphin über einem Stückchen dieses Metalls in einer Glasröhre und verändert sich 
weder das Camphin noch das Kalium, sondern bleibt dieses metallisch glänzend, so ist jenes rein. Bei dem geringsten Gehalte an Jod wird die Oberfäche des Kaliums blau angelaufen und es scheiden sich gelbliche Flocken aus dem Camphin ab. Auch Chlor ist ein sehr empfindliches Reagens auf Jod; leitet man nämlich dieses Gas in jodhaltiges Camphin, so färbt sich dieses sogleich schön rosenroth, wenn nur eine Spur Jod vorhanden war; bei bedeutenderm Gehalte an Jod wird das Camphin dunkelbraun. - Das reine Camphin ist ein farbloses, leichtflüssiges Oel, von angenehmem, dem Ol. Macis ähnlichen Geruche, der jedoch etwas terpentinähnlich ist. Sein spec. Gewicht ist bei $+25^{\circ} \mathrm{C}$. 0,827, sein Siedepunkt schwankt zwischen 167 bis $170^{\circ} \mathrm{C}$. bei 28". Es ist löslich in Alkohol, Aether, Terpentinöl und Petroleum, unlöslich in Wasser, schwachem Weingeiste, Kalilösung und verdünnten Säuren. Angezündet brennt es nit hellleuchtender, stark rufsender Flamme. Es destillirt sehr raseh und ohne die geringste Zersetzung. Von Schwefelsäure wird es nur wenig angegriffen. Mischt man es durchs Schütteln mit Schwefelsäurehydrat, so scheidet es sich bald wieder, ohne Veränderung erlitten zu haben, aus, nur ist die Säure etwas gelblich gefärbt. Rauchende Schwefelsäure wird etwas stärker vom Camphin gebräunt, als das Hydrat. Beim Erhitzen mit Schwefels. wird ein Theil Camphin zersetzt, die Sänre wird stark gefärbt, verdickt und entwickelt schwefelige Säure, jedoch destillirt ein Theil des Camphins über.

Salpetersäure im verdünnten Zustande wirkt sehr schwach auf Camphin ein, von rauchender Salpeters. wird es aber schon bei gewöhnlicher Temp. unter starkem Aufschäumen und Entwicklung von Stickstoffoxyd und salpetriger Säure oxydirt und dunkelroth gefärbt. Nach einiger Zeit verschwindet die Färbung und man erhält nach dem Waschen mit Wasser ein gelbes azothaltiges, nach Zimmt riechendes Oel. Lärst man längere Zeit hindurch rauchende Salpeters. auf Camphin ein- 
wirken, so verwandelt es sich in ein rothes dickes Oel, das sich in Aetzkali löst und diesem einen süfsen Geschmack ertheilt.

Concentrirte und verdünnte Salzs. wirken nicht bemerkbar auf das Camphin ein, und Chlorwasserstoffgas wird nur wenig absorbirt. 0,327 Gran Camphin über Quecksilber dem Einflusse des Salzsäuregases unterworfen, absorbirten innerhalb 4 Wochen nur 7,5 Centimeter jenes Gases. - Chlor äufsert auf Camphin eine starke Wirkung, wobei die Erscheinungen der Substitution wahrgenommen werden. Leitet man nämlich trocknes Chlor in Camphin, so wird unter starker Erhitzung und Bildung von Salzs. Chlor absorbirt. Das Camphin bildet unter Wasserstoffverlust Chlorverbindungen, welche viel Aehnlichkeit mit den Chlorkampherarten haben und nach Mafsgabe des absorbirten immer dickflüssiger werden, bis sie zuletzt so consistent geworden sind, dafs der Chlor nicht mehr einzuwirken vermag.

Brom wirkt so wie Chlor, nur heftiger auf Camphin ein. Giefst man Brom in kleinen Antheilen zum Camphin, so entsteht starkes Aufschäumen von Entwicklung der gebildeten Bromwasserstoffs. und die anfangs braun gefärbte Flüssigkeit wird nach einiger Zeit farblos. Das Camphin ist in Bromcamphin umgewandelt, das den Chlorcamphinen ganz ähnlich ist.

Jod löst sich leicht in Camphin auf. Geringe Mengen färben die Flüssigkeit schön rosenroth, gröfsere Antheile purpurviolett und dunkelbraun. Es scheint jedoch auf directem Wege kein Jodcamphin gebildet zu werden, da das Jod diese Substanz verharzt, indem sich Jodwasserstoffsäure bildet.

$$
\begin{array}{lrrrrr}
\multicolumn{7}{c}{\text { Analyse: }} \\
\text { C } & 87,30 & 87,29 & 87,05 & 87,24 & 87,38 . \\
\text { H } & 12,82 & 12,72 & 12,69 & 12,69 & 12,85 .
\end{array}
$$

entsprechend der Formel: $\mathrm{C}_{9} \mathrm{H}$ 16. Auch die Formel: C10 H 18 kommt dem Analysenresultate nahe, dann wäre das Camphin dem Menthen Walter's isomer.

Der Verf, zieht die erste Formel vor, weil für die 
rweite der Kohlenstoffgehalt nach der Analyse um ein Geringes zu hoch ausfällt. Daher pafst auch die Formel $\mathrm{C}_{18} \mathrm{H}_{32}$ besser für das Menthen, als die von Walter gewählte; denn er hat 87,5 C. erhalten.

Delalande hat einen Kohlenwasserstoff aus dem Kampher erhalten, den er Campholen nennt, welcher mit dem Camphin eine ganz gleiche Zusammensetzung hat und dessen Formel der Verf. auch $\mathrm{C}_{18} \mathrm{H}_{32}$ schreibt. Dieses Campholen siedet aber bei $+135^{\circ} \mathrm{C}$., während das Camphin erst bei $+176^{\circ} \mathrm{C}$. in's Kochen geräth. Auch das Menthen verhält sich in vielen Beziehungen dem Camphin analog.

Das Chlor entzieht dem Camphin, wie schon früher bemerkt worden, Wasserstoff und substituirt diesen, ohne seine neutrale Beschaffenheit aufzuheben. Die Chlorcamphine sind durchsichtige, olartige, farblose Körper von angenehm aromatischem, etwas terpentinähnlichem Geruche und kratzendem Geschmacke. Je gröfser ihr Gehalt an Chlor ist, desto consistenter sind sie. Sie lassen sich nicht ohne Zersetzung destilliren, sondern verhalten sich dabei wie die Chlorkampher und das Chlortereben.

In $10 \mathrm{Gr}$. Camphin wurde Chlor geleitet, das aus 4 Unzen Kochsalz entwickelt warde. Nach Beendigung der Operation wurde ein Strom trockner Luft durch die Verbindung gelassen, bis alles adhärirte Chlor und alle Salzsäure entfernt war. Das Product, von dem 18 Gr. erhalten wurden, war farblos, durchsichtig, von der Consistenz des Olivenöls, hatte bei $21^{\circ} \mathrm{C} .1,19$ spec. Gew., einen aromatischen, etwas terpentinähnlichen Geruch. Wird das Chlorcamphin in mit Kali gesättigtem Alkohol gelöst, so scheidet sich Chlorkalium aus und beim nochmaligen Vermischen der Lösung mit Wasser scheidet sich ein gelbes, sehr angenehm riechendes Oel ab, das weniger Chlor enthält, als das Chlorcamphin.

Die Analyse dieses Chlorcamphins gab folgende Resultate : 
Gräger: Ueber chromsaures Blei- und Zinkoxyd. 183

\begin{tabular}{ccccc} 
C & 47,2 & 47,3 & 18 & 48,00 \\
H & 5,57 & 5,63 & 26 & 5,65 \\
Cl & 46,70 & 46,7 & 6 & 46,35 \\
\hline
\end{tabular}

Wahrscheinlich ist jedoch nach dem Verhalten zum Kali zu urtheilen, die Formel folgende:

$\mathrm{C}_{18} \mathrm{H}_{24} \mathrm{Cl}_{4}+\mathrm{H}_{2} \mathrm{Cl}_{2}$.

Das Chlorcamphin wurde in einem mit trocknem Chlorgase angefüllten Glase dem Sonnenlichte und so lange ausgesetzt, bis kein Chlorgas mehr absorbirt wurde. Das Product war eine farblose, neutrale, klebrige Substanz von der Consistenz des venetianischeñ Terpentins, dem sie auch an Geruch ähnelt. Es gab bei der Analyse, auf 100 Theile, $32,87 \mathrm{C} 2,92 \mathrm{H}$ u. 64 Chlor. (Ans dem Bullet. scientif. de Pétersb. T. IX. p. 229 etc. vergl. Pharm. Centralbl. 1842, No, 6.)

\section{Ueber chromsaures Bleioxyd und chrom- saures Zinkoxyd; \\ N. $\stackrel{\text { von }}{\text { räger. }}$}

Hr. Dr. Boettger hat in seinen "Neuen Beiträgen zur Chemie und Physil « den Vorschlag gemacht, das in den Döbereiner'schen Platinfenerzeugen sich bildende schwefelsaure Zinkoxyd, nachdem man es vorher gereinigt, zur Darstellung von chromsaurem Zinkoxyd zu verwenden, welches Salz eine dem chromsauren Bleioxyd sehr ähnliche gelbe Farbe abyiebt, welche in manchen Fällen mit Vortheil das chromsaure Bleioxyd ersetzen könnte.

So zweckmäfsig ein solches Verfahren auch beim ersten Anblick erscheint, so erweist es sich doch bei näherer Prüfung als höchst unvortheilhaft. Man braucht nur, um dies einzusehen, die Atomenzahlen des chromsauren Kalis, des chromsauren Bleioxyds und Zinkoxyds, so wie die des schwefelsauren Zinkoxyds und essigsauren Bleioxyds unter sich za vergleichen. 